\title{
Force rebalance control for a MEMS gyroscope using ascending frequency drive and generalized PI control
}

\author{
Chun-Hua HE ${ }^{1,2}$, Qin-Wen HUANG ${ }^{2}$, Qian-Cheng ZHAO ${ }^{1}$, Zhen-Chuan YANG ${ }^{1}$, Da-Cheng ZHANG ${ }^{1}$ and Gui-Zhen YAN ${ }^{1}$ \\ ${ }^{1}$ National Key Laboratory of Science and Technology on Micro/Nano Fabrication, Institute of Microelectronics, Peking University, Beijing \\ 100871, P. R. China \\ ${ }^{2}$ Science and Technology on Reliability Physics and Application of Electronic Component Laboratory, No.5 Electronics Research Institute of \\ the Ministry of Industry and Information Technology, Guangzhou, 510610, Guangdong, P. R. China
}

\begin{abstract}
This paper has proposed a novel force rebalance control method for a MEMS gyroscope using ascending frequency drive and generalized PI control. Theoretical analyses of ascending frequency drive and force rebalance control methods are illustrated in detail. Experimental results demonstrate that the electrical anti-resonant peaks are located at the frequency responses in the RFD system, which seriously deteriorates the original response characteristics. However, they are eliminated in the AFD system, and the electrical coupling signal is also suppressed. Besides, as for the force rebalance control system, the phase margins approximate to $60 \mathrm{deg}$, gain margins are larger than $13 \mathrm{~dB}$, and sensitivity margins are smaller than $3.2 \mathrm{~dB}$, which validates the control system is stable and robust. The bandwidth of the force rebalance control system is measured to be about $103.2 \mathrm{~Hz}$, which accords with the simulation result. The bias instability and angle random walk are evaluated to be $1.65 \mathrm{deg} / \mathrm{h}$ and $0.06 \mathrm{deg} / \sqrt{\mathrm{h}}$, respectively, which achieves the tactical level.
\end{abstract}

Keywords: MEMS gyroscope, mode-matching, force rebalance control, ascending frequency drive, electrical coupling suppressing.

\section{Introduction}

Electrical coupling is one of the major error sources for a MEMS gyroscope, which is induced by the direct coupling from the drive combs to the sense combs through the parasitic capacitances [1-2]. It will bring about the non-ideal anti-resonance at the amplitude-frequency responses of drive mode and sense mode, which deteriorates the performance and affects the reliability of the closed loop system. Therefore, it is significant to suppress the electrical coupling signals by modifying the control and readout circuit. Electromechanical amplitude modulation (EAM) [3] method can modulate the actual vibration signal by exerting a high-frequency carrier (usually several $\mathrm{MHz}$ ) to the public mass. Thus, coupling signals can be separated and eliminated from the useful sense signal easily. However, a demodulation process should be added to restore the useful information, which will complicate the control system. Furthermore, the circuit has to handle the demodulation in high frequency domain, which increases the hardware cost, power consumption and high frequency noise.

By introducing a drive modulation process, ascending frequency drive (AFD) method [4-5] is proved effective to suppress the electrical coupling for the drive mode of a MEMS gyroscope. Compared with EAM, it can simplify the readout circuit since it needs no extra demodulation process. In addition, compared to resonant frequency drive (RFD) method, the noise in AFD system can be further suppressed. Hence, in this paper, AFD method will be applied to the force rebalance control system for sense mode of a MEMS gyroscope. Nowadays, force rebalance control for the sense mode is widely adopted in high-performance gyroscopes, since it can adjust the bandwidth, improve the nonlinearity, extend the measure range [6-9]. As for EAM 
method, a lot of researches about the force rebalance control have been fulfilled and reported in our previous work [8-9]. In this work, novel theoretical analysis of force rebalance control with AFD method will be conducted in detail.

\section{Theoretical analysis}

\section{Electrical coupling suppressing}

The simplified schematic of a Z-axis doubly decoupled MEMS tuning fork gyroscope is shown in Fig. 1 (a). It mainly includes drive combs, drive-sensing combs, sense combs, force feedback combs, and mode-matching combs. The key parameters of the tested gyroscope at room temperature are listed in Table 1. The resonant frequency of the sense mode $\omega_{s}$ will equal the resonant frequency of the drive mode $\omega_{d}$ after mode-matching control. Fig. 1 (b) shows that there are some parasitic capacitances between the drive combs and sense combs since they are close to each other in the layout.

Table 1. Key parameters of the tested gyroscope at room temperature.

\begin{tabular}{cc}
\hline \hline Parameter & Value \\
\hline Drive mode resonant frequency $f_{d}$ & $9451.5 \mathrm{~Hz}$ \\
Drive mode quality factor $Q_{d}$ & 11385 \\
Sense mode resonant frequency $f_{s}$ & $9481.1 \mathrm{~Hz}$ \\
Sense mode quality factor $Q_{s}$ & 2300 \\
\hline \hline
\end{tabular}

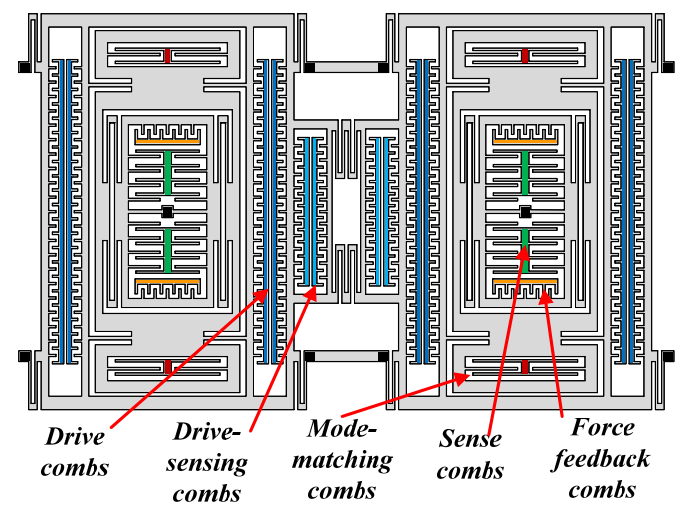

(a) The simplified schematic of a Z-axis doubly decoupled MEMS tuning fork gyroscope

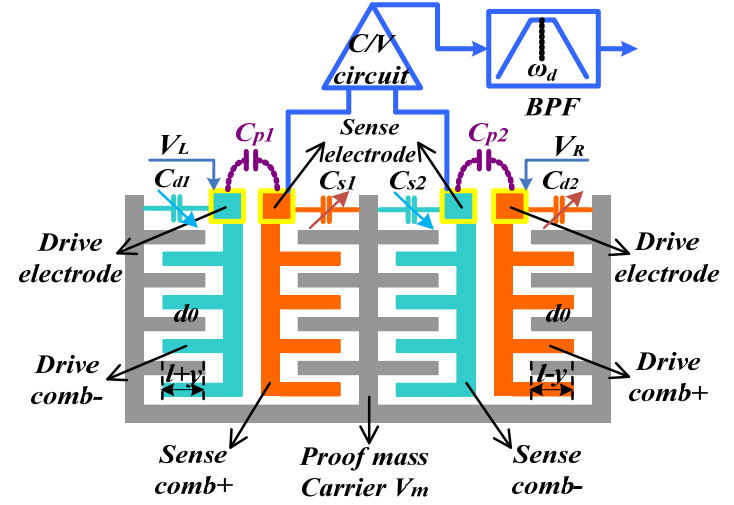

(b) Electrical coupling analysis between the drive combs and sense combs of a MEMS gyroscope

Fig. 1. Electrical coupling analysis of a MEMS gyroscope.

In order to fulfill the differential detection, a $D C$ carrier signal $V_{m}$ is exerted to the proof mass. $C_{d 1}$ and $C_{d 2}$ are the differential drive capacitances, while $C_{s 1}$ and $C_{s 2}$ are the differential sense capacitances. $C_{p 1}$ and $C_{p 2}$ stand for the differential parasitic capacitances between the drive combs and sense combs. $V_{L}$ and $V_{R}$ represent the two differential drive signals. Owing to the existence of parasitic capacitances, drive signals can be coupled to the sense combs, which will disturb the original detection signal and deteriorate the closed loop system's stability and performance. Fig. 2 and Fig. 3 depict the schematics of the readout circuit and AFD circuit for the sense mode of a MEMS gyroscope, respectively. In the readout circuit, the output of differential amplifier can be derived as:

$$
V_{\text {out }}=\frac{k_{g}\left[V_{m}\left(C_{s 2}-C_{s 1}\right)+\left(V_{R} C_{p 2}-V_{L} C_{p 1}\right)\right]}{C_{f}}
$$

Where, $k_{g}$ is the gain of the differential amplifier, and $C_{f}$ is the feedback capacitance of the charge amplifier. In the RFD circuit, the frequencies of $V_{L}$ and $V_{R}$ equals to those of $C_{s I}$ and $C_{S 2}$, thus it is hard to distinguish $V_{m}\left(C_{s 2}-C_{s 1}\right)$ from $\left(V_{R} C_{p 2}-V_{L} C_{p 1}\right)$. Due to the influence of coupling signal, there will be a non-ideal anti-resonance appeared at the amplitude-frequency response [4-5]. However, in the AFD circuit, the frequency of $V_{L}$ and $V_{R}$ is far larger than that of $C_{s 1}$ and $C_{S 2}$, thus the coupling signal $\left(V_{R} C_{p 2}-V_{L} C_{p 1}\right)$ can be eliminated by a suitable band-pass-filter (BPF). 
Therefore, after filtered by BPF, $y_{\text {out }}$ can be described as:

$$
y_{\text {out }}=\frac{k_{g} V_{m}\left(C_{s 2}-C_{s 1}\right)}{C_{f}}=\frac{2 k_{g} V_{m} N_{s} \varepsilon h}{C_{f} d_{0}} y
$$

Where, $y$ is the displacement of the sense mode, $\varepsilon$ is the permittivity, $N_{s}$ is the number of sense capacitances, $h$ is the thickness, and $d_{0}$ is the gap. From equation (2), the gain $k_{c v}$ of the readout circuit can be obtained as:

$$
k_{c v}=\frac{2 k_{g} V_{m} N_{s} \varepsilon h}{C_{f} d_{0}}
$$

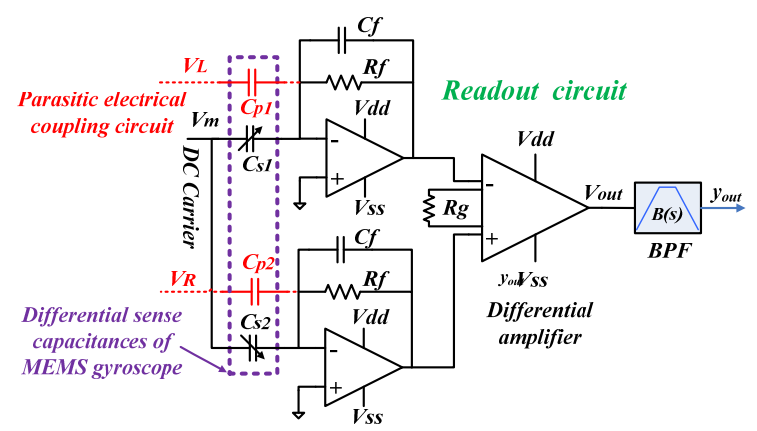

Fig. 2. The readout circuit for the sense mode of a MEMS gyroscope.

$$
\begin{aligned}
& V_{2}=V_{R}-V_{m}+n_{2}=\left(V_{d c}+V_{a c} \sin \left(\omega_{d} t\right)\right) P \sin \left(\omega_{c} t\right)-V_{m}+n_{2} \\
& V_{1}=V_{L}-V_{m}+n_{1}=\left(V_{d c}-V_{a c} \sin \left(\omega_{d} t\right)\right) P \sin \left(\omega_{c} t\right)-V_{m}+n_{1}
\end{aligned}
$$

From Fig. 1 (b), the energies of the differential drive capacitances can be expressed as:

$$
\begin{gathered}
E_{2}=\frac{N V_{2}^{2} \varepsilon h(l-y)}{d_{0}} \quad \begin{array}{c}
\text { Where, } N \text { is the number of drive } \\
\text { capacitances, and } l \text { is the overlapped length. } \\
\text { Then, the total drive force can be derived as: }
\end{array} \\
F_{t o t}=\frac{-\partial E_{1}}{\partial y}+\frac{-\partial E_{2}}{\partial y}=\frac{N \varepsilon h\left(V_{2}^{2}-V_{1}^{2}\right)}{d_{0}}=\frac{N \varepsilon h}{d_{0}}\left[2 P^{2} V_{d c} V_{a c} \sin \left(\omega_{d} t\right)+\left(n_{2}+n_{1}-2 V_{m}\right)\left(n_{2}-n_{1}\right)\right. \\
\left.-2 P^{2} V_{a c} V_{d c} \sin \left(\omega_{d} t\right) \cos \left(2 \omega_{c} t\right)+2\left(n_{2}-n_{1}\right) V_{d c} P \sin \left(\omega_{c} t\right)+2\left(n_{2}+n_{1}-2 V_{m}\right) V_{a c} P \sin \left(\omega_{d} t\right) \sin \left(\omega_{c} t\right)\right]
\end{gathered}
$$

Since the frequency response of a gyroscope can be regarded as a BPF, the lower and higher frequency components will be suppressed, and the real useful force can be yielded as:

$$
F_{\text {useful }}=\frac{2 N \varepsilon h P^{2} V_{d c}}{d_{0}} V_{a c} \sin \left(\omega_{d} t\right)
$$

Equation (11) indicates that the noises can be suppressed, and there is no need to demodulate the readout signal $y_{\text {out }}$ since the frequency of $y$ is $\omega_{d}$, rather than $\omega_{c} \pm \omega_{d}$. Hence, the gain $k_{v f}$ of the drive circuit can be expressed as:

$$
k_{v f}=\frac{2 N \varepsilon h P^{2} V_{d c}}{d_{0}}
$$

\section{Force rebalance control}

AFD method is effective to suppress the coupling signal, which is detailedly illustrated above. $k_{c v}$ and $k_{v f}$ of the AFD circuit are yielded in equations (3) and (12). Thus the force 
rebalance control system for the sense mode can be introduced as Fig. 4. Where, $-\Omega(t)$ and $q(t)$ are input angular rate and quadrature coupling signal, respectively. $F_{c}, F_{q}$ and $F_{b}$ represent Coriolis force, quadrature force, and feedback force, respectively. $m_{p} A_{v}, k_{q f}$ and $k_{v f}$ are corresponding force coefficients of them. $m_{s}$ and $m_{p}$ represent the sense mass and proof mass, respectively. $A_{v}$ stands for the amplitude of the drive velocity. $k_{d c}$ is the conversion coefficient from displacement to capacitance, and $\theta$ is the demodulation phase. The transfer functions of the sense mode $G_{s}(s)$, BPF $B(s)$, low-pass-filter (LPF) $L(s)$, and generalized-PI (G-PI) controller $P(s)$ are defined as follows:

$$
\begin{aligned}
& G_{s}(s)=\frac{k_{d c} / m_{s}}{s^{2}+s \omega_{s} / Q_{s}+\omega_{s}^{2}} \\
& B(s)=\frac{2 \xi_{b} \omega_{b} s}{s^{2}+2 \xi_{b} \omega_{b} s+\omega_{b}^{2}} \\
& L(s)=\frac{\omega_{l}^{2}}{s^{2}+2 \xi_{l} \omega_{l} s+\omega_{l}^{2}} \\
& P(s)=\frac{k_{p}\left(s+\omega_{2}\right)}{\left(s+\omega_{1}\right)\left(s+\omega_{3}\right)}
\end{aligned}
$$

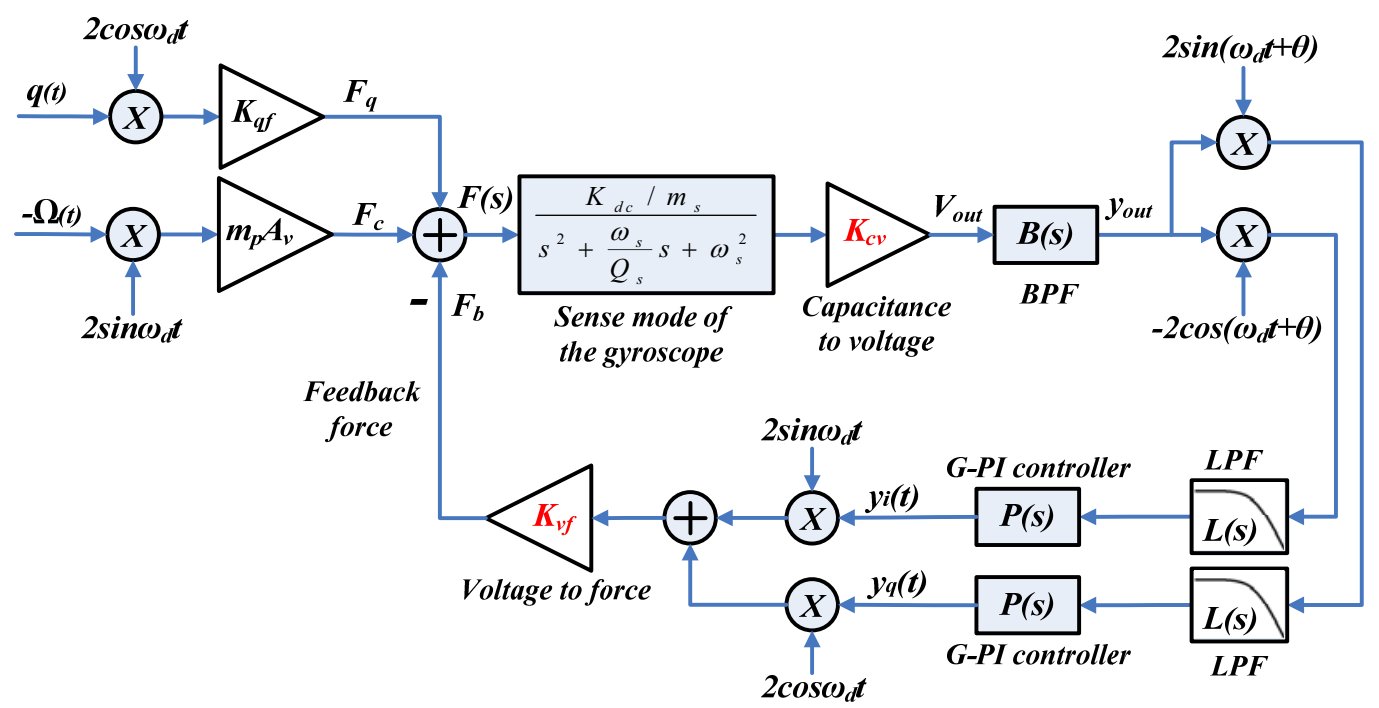

Fig. 4. Force rebalance control system with AFD method for the sense mode of a MEMS gyroscope.

Where, $\xi_{b}$ and $\omega_{b}$ are the damping ratio and centre frequency of BPF, $\xi_{l}$ and $\omega_{l}$ are the damping ratio and cut-off frequency of LPF, $k_{p}$, $\omega_{1}, \omega_{2}$ and $\omega_{3}$ are the key parameters of G-PI controller. There are two control loops shown in Fig. 4, one is utilized to rebalance the
Coriolis force, while another is used to rebalance the quadrature force. As for this force control system, the transfer function of the open loop system $D(s)$ can be deducted easily based on Fig. 4:

$$
D(\mathrm{~s})=\frac{F_{b}(s)}{F(s)}=2 j k_{c v} k_{v f}\left[e^{-j \theta} L\left(j \omega-j \omega_{d}\right) P\left(j \omega-j \omega_{d}\right)-e^{j \theta} L\left(j \omega+j \omega_{d}\right) P\left(j \omega+j \omega_{d}\right)\right] B(j \omega) G_{s}(j \omega)
$$

Where, complex frequency $s$ is equal to $j \omega$. Thus the transfer function of the closed loop system $T(s)$ and the sensitivity function $S(S)$ can be yielded as:

$$
S(s)=\frac{1}{1+D(s)}
$$

$$
T(s)=\frac{D(s)}{1+D(s)}
$$




\section{Experimental test}

\section{Swept frequency test}

The simplified schematic of a Z-axis doubly decoupled MEMS tuning fork gyroscope is shown in Fig. 5. It mainly includes an analog circuit and a digital circuit. Swept frequency tests of the sense mode are conducted in the RFD system and AFD system, respectively. Experimental results demonstrate that the electrical anti-resonant peaks are located at the amplitude-frequency and phase-frequency responses in the RFD system, which seriously deteriorates the original response characteristics, as shown in Fig. 6 (a). Due to the existence of electrical anti-resonant peaks, it is very hard to design a suitable G-PI controller to guarantee the reliability and robustness of the force rebalance control system. Therefore, it is urgent to suppress the electrical coupling. On the other hand, in Fig. 6 (b), there are no anti-resonant peaks appeared in the AFD system, and the electrical coupling signal is eliminated, which indicates the theoretical analysis is accurate.

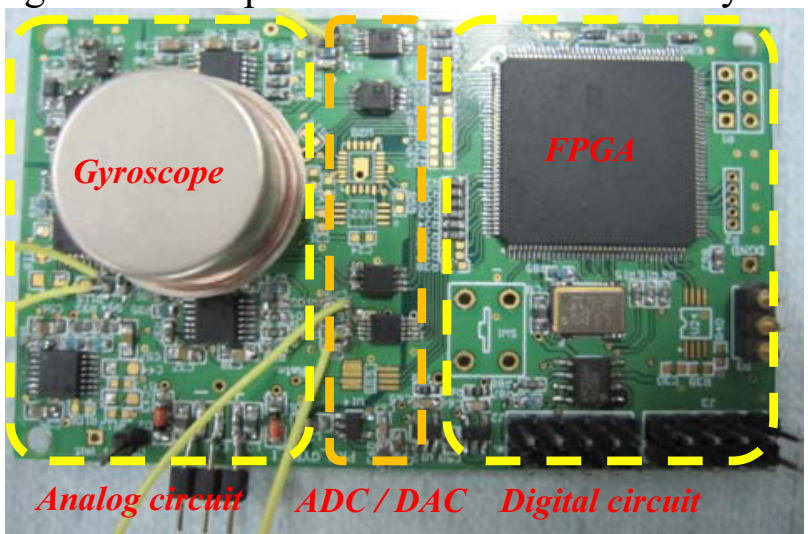

Fig. 5. The simplified schematic of a Z-axis doubly decoupled MEMS tuning fork gyroscope.

\section{Force rebalance control test}

After the theoretical deduction above, equations (3) and (12)-(19) can be utilized to design the closed loop system. Simultaneously, simulations based on these equations are accomplished with Matlab software, as shown in Fig. 7 and Fig. 8. In Fig. 7, owing to narrow-band force rebalance control, there are two crossover frequencies appearing in the frequency-amplitude response of $D(s)$. Thus, similar to our previous analysis [9], there are two corresponding phase margins $\left(P M_{l}\right.$ and $\left.P M_{r}\right)$, gain margins $\left(G M_{l}\right.$ and $\left.G M_{r}\right)$, cut-off frequencies $\left(f_{t l}\right.$ and $\left.f_{t r}\right)$ and sensitivity margins $\left(M_{s l}\right.$ and $\left.M_{s r}\right)$, as marked in the figure. The key stability indices of the feedback control system obtained by Matlab simulation are listed in Table 2. According to classical linear control theory, the force rebalance control system is stable and robust enough. Besides, the bandwidth $\left(B W_{l r}\right)$ of $T(s)$ is calculated to be $103.18 \mathrm{~Hz}$, which means the force outside this narrow-band will never be balanced.

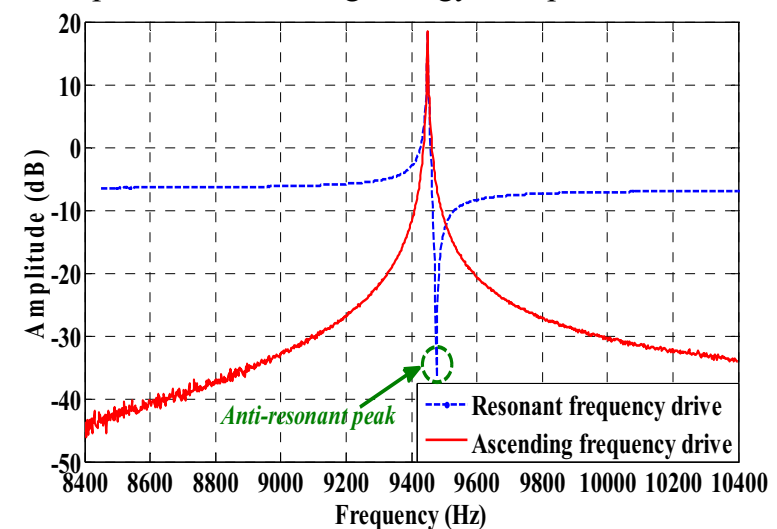

(a) Amplitude-frequency response

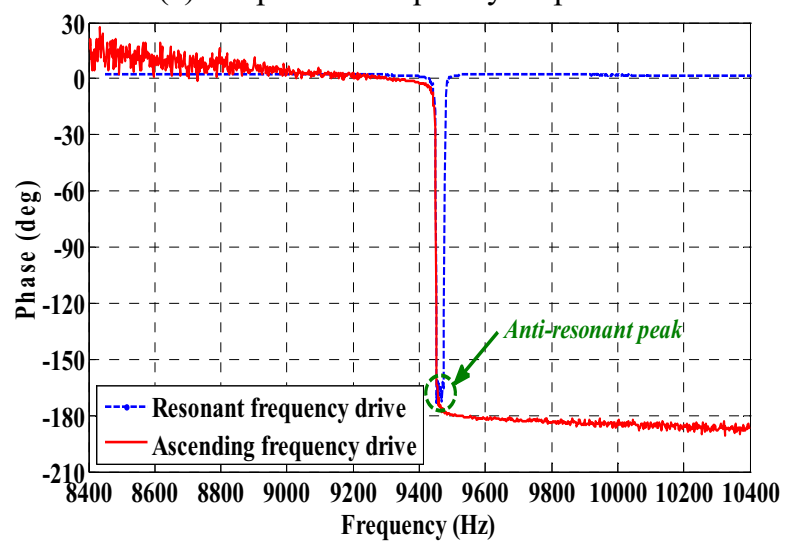

(b) Phase-frequency response 
Fig. 6. Swept frequency tests of the sense mode in RFD

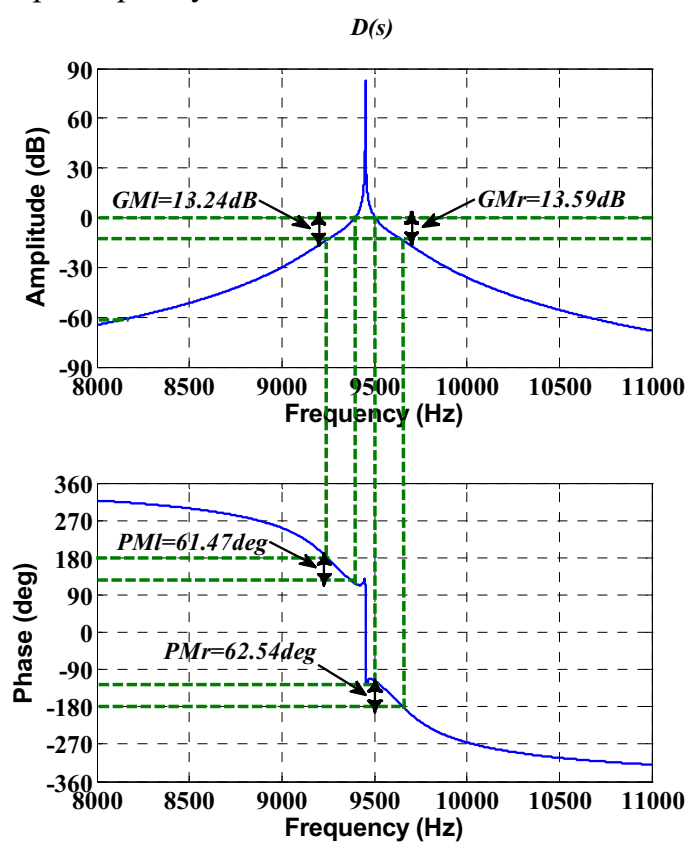

and AFD methods.

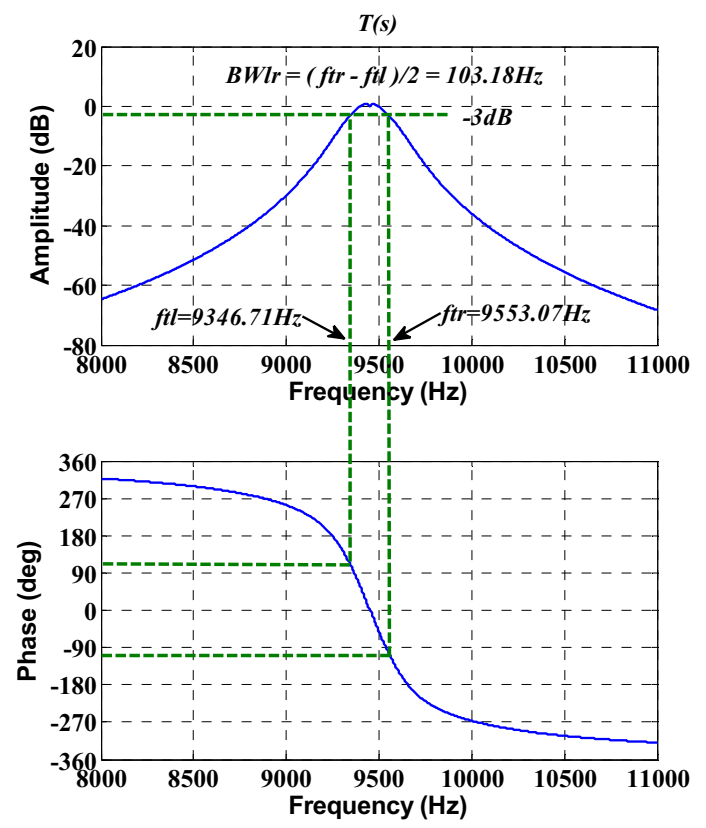

Fig. 7. The frequency responses of the open loop system $D(s)$ and closed loop system $T(s)$.

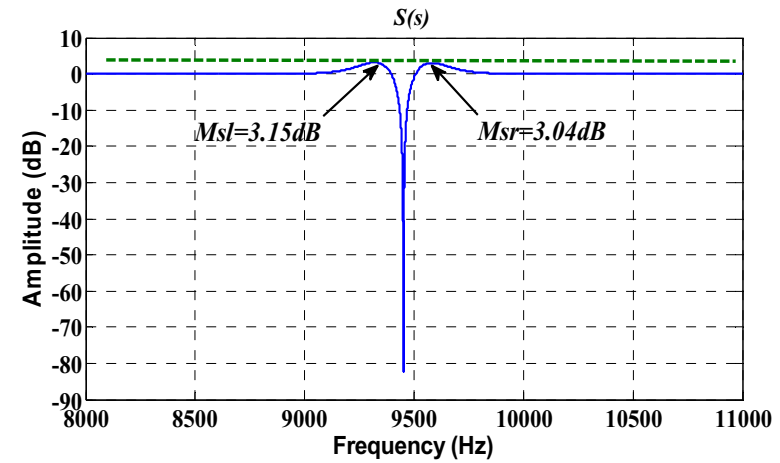

Fig. 8. The frequency response of the sensitivity function $S(s)$.

Table 2. Key stability indices of the feedback control system obtained by Matlab simulation.

\begin{tabular}{ccc}
\hline Type & \multicolumn{2}{c}{ Key stability indices } \\
\hline Phase margin & $P M_{l}=61.47$ & $P M_{r}=62.54$ \\
PM (deg) & & \\
Gain margin & $G M_{l}=13.24$ & $G M_{r}=13.59$ \\
GM (dB) & & \\
Sensitivity & $M_{s l}=3.15$ & $M_{s r}=3.04$ \\
margin Ms (dB) & & \\
Cut-off & $f_{t l}=9346.71$ & $f_{t r}=9553.07$ \\
frequency $f_{t}(\mathrm{~Hz})$ & & \\
Bandwidth BW & $B W_{l r}=\left(f_{t r}-f_{t l}\right) / 2=103.18 \mathrm{~Hz}$ \\
\hline$(\mathrm{Hz})$ &
\end{tabular}

The bandwidth (BW) of the force rebalance control system is measured to be about 103.2Hz, which approximates to the simulation result, as shown in Fig. 9. It further verifies the theoretical analysis of the closed loop system is precise. The scale factor of the MEMS gyroscope with closed loop controlled sense mode is $65.9 \mathrm{mV} / \mathrm{deg} / \mathrm{s}$ with nonlinearity of $0.03 \%$. Fig. 10 illustrates that the bias instability and angle random walk are evaluated to be $1.65 \mathrm{deg} / \mathrm{h}$ and $0.06 \mathrm{deg} / \sqrt{\mathrm{h}}$, respectively, which reaches the tactical level. 


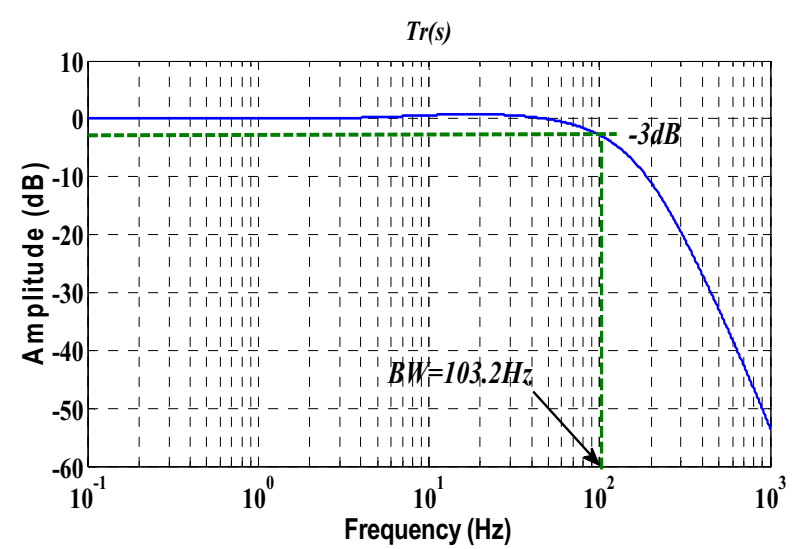

Fig. 9. Bandwidth test results of the closed loop control system.

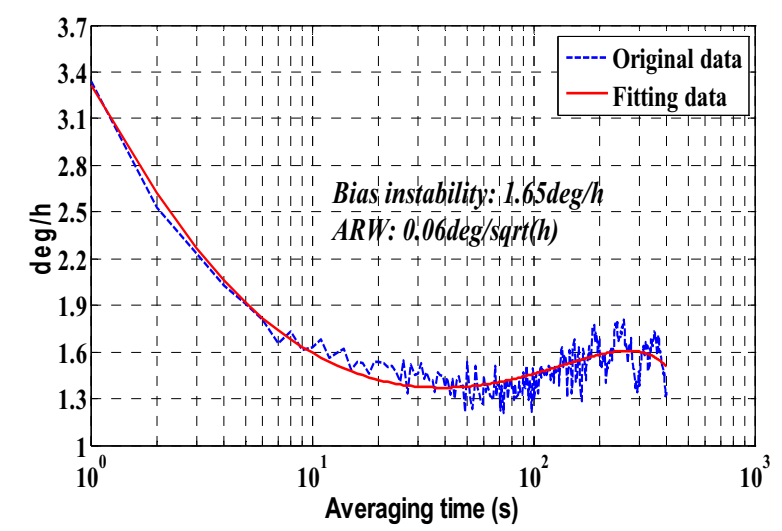

Fig. 10. Allan variance graph of bias drift of the MEMS gyroscope with closed loop controlled sense mode.

\section{Summary}

In this paper, a novel force rebalance control method has been presented for a MEMS gyroscope using ascending frequency drive and generalized PI control. Theoretical analyses of ascending frequency drive and force rebalance control methods are illustrated in detail. Experimental results demonstrate that the electrical anti-resonant peaks are located at the amplitude-frequency and phase-frequency responses in the RFD system, which seriously deteriorates the original response characteristics. However, they are eliminated in the AFD system, and the electrical coupling signal is also suppressed. On the other hand, the frequency responses of the force rebalance control system indicate that the phase margins approximate to $60 \mathrm{deg}$, gain margins are larger than $13 \mathrm{~dB}$, and sensitivity margins are smaller than $3.2 \mathrm{~dB}$, which validates the control system is stable and robust. The bandwidth of the force rebalance control system is measured to be about $103.2 \mathrm{~Hz}$, which verifies the theoretical analysis is accurate. The bias instability and angle random walk are evaluated to be $1.65 \mathrm{deg} / \mathrm{h}$ and $0.06 \mathrm{deg} / \sqrt{\mathrm{h}}$, respectively, which reaches the tactical level.

\section{Acknowledgement}

This work is partially supported by the National Natural Science Foundation of China under Grant No. 61434003.

\section{References}

[1] Lee, H. Ko, et al., "Non-ideal behavior of a driving resonator loop in a vibratory capacitive microgyroscope", Microelectronics J., vol. 39, 2008, pp. 1-6.

[2] M.S. Weinberg and A. Kourepenis, "Error sources in in-plane silicon tuning-fork MEMS gyroscopes", J. Microelectromech. Syst., vol. 15, 2006, pp. 479-491.

[3] Acar, A.M. Shkel, "Structurally decoupled micromachined gyroscopes with post-release capacitance enhancement", J. Micromech. Microeng., 2005, 15 1092-1101.

[4] J. Cui, Z. Y. Guo, Z.C. Yang, et al., "Electrical coupling suppressing for a microgyroscope using ascending frequency drive with 2-DOF PID controller", Proc. Transducers 2011, pp. 2002-2005.

[5] J. Cui, Z.Y. Guo, Z.C. Yang, et al., "Electrical coupling suppression and transient response improvement for a microgyroscope using ascending frequency drive with a 2-DOF PID controller", J. Micromech. Microeng., 2011, 21095020.

[6] S. Sonmezoglu, S.E. Alper, T. Akin, "An Automatically Mode-Matched MEMS Gyroscope With Wide and Tunable Bandwidth", J. Microelectromech. Syst., Vol. 23, No. 2, 2014, pp.284-297.

[7] R. Mirjalili, H. Wen, D. E. Serrano, and F. Ayazi, "Substrate-decoupled silicon disk resonant having degenerate gyroscopic 
modes with Qin excess of 1-million", Proc.

TRANSDUCERS 2015, pp. 15-18.

[8] C.H. He, Q.C. Zhao, Y.X. Liu, et al., "Closed loop control design for the sense mode of micromachined vibratory gyroscopes", Sci. China Tech. Sci. 2013, Vol. 56, pp. 1112-1118.

[9] C.H. He, D.C. Liu, Q.C. Zhao, et al., "A novel narrow-band force rebalance control method for the sense mode of MEMS vibratory gyroscopes", Measurement, vol. 62, 2015, pp. 197-204. 